\title{
A numerical study of a method for measuring the effective in situ sound absorption coefficient
}

\author{
Erwin R. Kuipers, ${ }^{\text {a) }}$ Ysbrand H. Wijnant, and André de Boer \\ Chair of Structural Dynamics and Acoustics, Faculty of Engineering Technology \\ University of Twente, Enschede, The Netherlands \\ e.r.kuipers@ctw.utwente.nl,y.h.wijnant@ctw.utwente.nl,a.deboer@ctw.utwente.nl
}

\begin{abstract}
The accuracy of a method [Wijnant et al., Proc. of ISMA 31, Leuven, Belgium (2010), Vol. 31] for measurement of the effective areaaveraged in situ sound absorption coefficient is investigated. Based on a local plane wave assumption, this method can be applied to sound fields for which a model is not available. Investigations were carried out by means of finite element simulations for a typical case. The results show that the method is a promising method for determining the effective areaaveraged in situ sound absorption coefficient in complex sound fields.
\end{abstract}

(C) 2012 Acoustical Society of America

PACS numbers: 43.55.Ev, 43.55.Dt, 43.20.El, 43.20.Ye [NX]

Date Received: May 22, 2012 Date Accepted: July 31, 2012

\section{Introduction}

Measurement of the in situ sound absorption coefficient is hardly possible in small enclosures, such as cars or small rooms. This difficulty originates from the definition of the in situ sound absorption coefficient: It is the sound absorption coefficient for a specified angle of incidence for the in situ structure in a semi-anechoic field. See, for example, Brandão et al. ${ }^{1}$ Accordingly, in situ sound absorption measurement methods are intended for measurements in semi-anechoic fields. ${ }^{1-5}$ Exceptions are methods that employ a time-windowing technique ${ }^{6-8}$ However, in small enclosures, measurements at low frequencies cannot be performed as the length of the time window needs to be very short to gate out spurious reflections.

A measurement concept that is likely to function well in small enclosures is the method described by Takahashi et al. ${ }^{9}$ Here, the normal surface acoustic impedance is measured while the sample is exposed to environmental ambient noise. Otsuru et al. ${ }^{10}$ and Din et al. ${ }^{11}$ analyzed this method in more detail, and showed that accurate results can be obtained, even at low frequencies. Otsuru et al. conclude that ensemble averaging over multiple spatially distributed incoherent sources is an effective means of reducing the undesirable effects of edge-diffracted waves and other interfering waves.

Instead, one could consider the concept of area averaging to reduce the aforementioned effects. This is the approach followed by the measurement method discussed in this paper, as first presented by Wijnant et al. ${ }^{12}$ This method is based on a local plane wave assumption, and is therefore hereafter referred to as the LPW method. This method is devised for use in stationary sound fields, and generally yields the effective in situ sound absorption coefficient, being an indicator of the sound absorbing performance of an in situ structure in the actual sound field. Although the effective area-averaged in situ absorption coefficient is not directly comparable with laboratory coefficients, we believe that it is of practical value. Purposes are quality control or the evaluation of the absorbing performance of an absorber in the actual sound field, for instance if room-acoustic performance indicators are out of specification.

In this paper, the accuracy of the LPW method is investigated. To this purpose, we performed an acoustic finite element analysis to simulate the determination of the effective

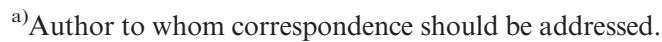


area-averaged sound absorption coefficient for a sound field with multiple reflections. Influences of the distance of the measurement surface to the surface of interest, the size of the surface area, and the surface impedance were investigated. The results are compared with those obtained with an existing measurement method. The theory of the LPW method is introduced in Sec. 2. The results of the investigations are presented and discussed in Sec. 3.

\section{Theory}

In this section, we introduce the LPW method through a concise presentation of the theory given by Wijnant et al. ${ }^{12}$ The LPW method has been devised for stationary sound fields, and therefore the theory will be shown for the case of pure tones. The objective of the LPW method is to determine the effective area-averaged sound absorption coefficient $\alpha_{\mathrm{LPW}}(\omega)$ of a measurement surface $S^{\prime}$ at a distance $d$ from the material surface $S_{m}$, see Fig. 1(a). It is reasonable to assume that this coefficient is an accurate estimate if $d$ is sufficiently small. Therefore, it is not necessary to have an overall sound field model with which the surface impedance at the material surface can be calculated.

$\alpha_{\text {LPW }}(\omega)$ is defined as the ratio of the active and incident sound power with respect to surface $S^{\prime}$,

$$
\alpha_{\mathrm{LPW}}(\omega)=\frac{\int_{S^{\prime}} \mathbf{I}_{\mathrm{ac}}(\mathbf{r}, \omega) \cdot \mathbf{n} d S}{\int_{S^{\prime}} I_{\mathrm{in}}(\mathbf{r}, \omega) d S},
$$

where $\mathbf{I}_{\mathrm{ac}}(\mathbf{r}, \omega)$ is the active sound intensity vector, $I_{\mathrm{in}}(\mathbf{r}, \omega)$ is the incident sound intensity perpendicular to the surface $S^{\prime}$, vector $\mathbf{r}$ indicates the spatial position, $\omega$ is the radial frequency, and the direction vector $\mathbf{n}=-\mathbf{n}_{S}$, where $\mathbf{n}_{S}$ is the surface normal. Determination of the active sound power is possible by using existing sound intensity measurement techniques, ${ }^{13,14}$ and is therefore not discussed in this paper. It is the determination of the incident power that is addressed by the LPW method. To this purpose, we formulated the local plane wave assumption, from which the LPW method inherited its name: In any point on $S^{\prime}$, its location defined by $\mathbf{r}$, and in direction $\mathbf{n}$, we locally approximate the sound field by an incident plane wave with complex amplitude $A(\mathbf{r}, \omega)$ traveling toward $S_{m}$, and an oppositely directed reflected plane wave with complex amplitude $B(\mathbf{r}, \omega)$. According to this assumption, the sound pressure and particle velocity in direction $\mathbf{n}$ can be written as

$$
P(\mathbf{r}, x, \omega)=A(\mathbf{r}, \omega) e^{-i k x}+B(\mathbf{r}, \omega) e^{i k x},
$$

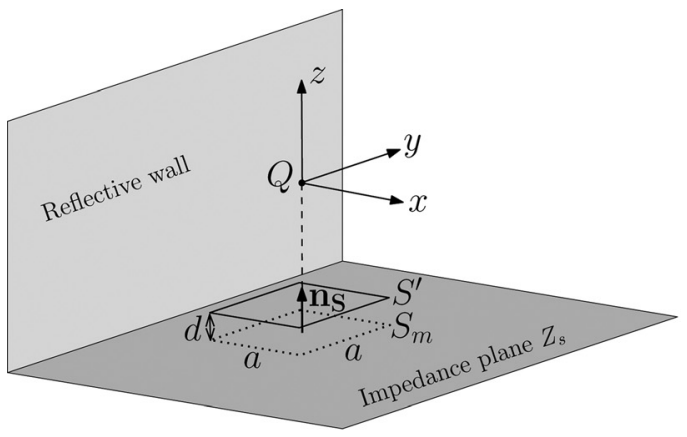

(a) Configuration

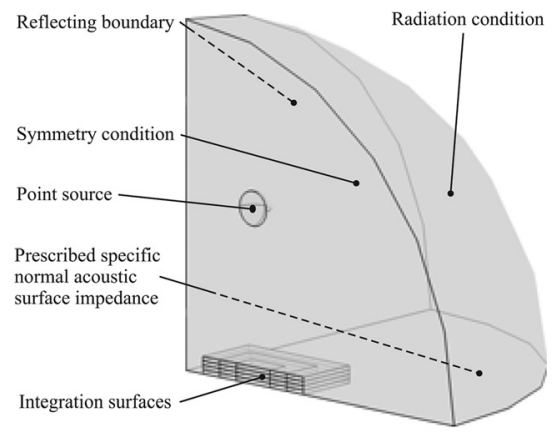

(b) FE-model

Fig. 1. Configuration and finite element model: A point source $Q$ is located at height $h=1 \mathrm{~m}$ above a semiinfinite impedance plane. A square section with side length $a$ of this plane is defined as the material surface $S_{m}$. The measurement surface $S^{\prime}$ also has side length $a$, and has a distance $d$ to $S_{m}$. A semi-infinite reflective wall is located at $x=-0.5 \mathrm{~m}$. 


$$
\mathbf{U}(\mathbf{r}, x, \omega) \cdot \mathbf{n}=\frac{1}{\rho_{0} c_{0}}\left[A(\mathbf{r}, \omega) e^{-i k x}-B(\mathbf{r}, \omega) e^{i k x}\right],
$$

where $x$ is a spatial coordinate aligned with $\mathbf{n}, \rho_{0}$ is the density, $c_{0}$ is the speed of sound, and $k$ is the wavenumber. Setting $x=0$ at the point of consideration and solving for both $A(\mathbf{r}, \omega)$ and $B(\mathbf{r}, \omega)$ yields

$$
\begin{aligned}
& A(\mathbf{r}, \omega)=\frac{1}{2}\left[P(\mathbf{r}, \omega)+\rho_{0} c_{0} \mathbf{U}(\mathbf{r}, \omega) \cdot \mathbf{n}\right], \\
& B(\mathbf{r}, \omega)=\frac{1}{2}\left[P(\mathbf{r}, \omega)-\rho_{0} c_{0} \mathbf{U}(\mathbf{r}, \omega) \cdot \mathbf{n}\right] .
\end{aligned}
$$

$A(\mathbf{r}, \omega)$ and $B(\mathbf{r}, \omega)$ generally vary with position. Associating the incident sound intensity with the incident wave, the incident sound intensity becomes

$$
I_{\text {in.n, }, \mathrm{LPW}}(\mathbf{r}, \omega)=\frac{|A(\mathbf{r}, \omega)|^{2}}{2 \rho_{0} c_{0}} .
$$

The reflected sound intensity can be determined by replacing $A(\mathbf{r}, \omega)$ by $B(\mathbf{r}, \omega)$ in Eq. (6). After completion of a series of point measurements, the incident sound intensity distribution over $S^{\prime}$ is obtained. Together with the distribution of the active sound intensity, the effective in situ area-averaged sound absorption coefficient $\alpha_{\mathrm{LPW}}(\omega)$ is calculated with Eq. (1). This coefficient becomes a regular in situ sound absorption coefficient for measurements in semi-anechoic sound fields where plane waves are normally incident upon a locally reacting surface.

\section{Investigation of the accuracy of the LPW method}

In this section, the accuracy of the LPW method is investigated by applying an acoustic finite element analysis for the configuration shown in Fig. 1(a). This configuration is chosen for two reasons: (1) A measurement with existing methods ${ }^{1-8}$ is not possible for this configuration and (2) the incident intensity can be calculated analytically so that the accuracy of the LPW method can be evaluated. The LPW method is applied to estimate the effective area-averaged sound absorption coefficient of the square surface section $S_{m}$. The surface impedance $Z_{S}$, the side length $a$, and the distance $d$ are varied to study their effect on the accuracy.

To demonstrate what happens if a semi-anechoic measurement method is applied to the (non-semi-anechoic) sound field studied here, we have also calculated the effective sound absorption coefficient with an existing in situ method, the twomicrophone method by Allard and Sieben. ${ }^{2}$ This calculation is performed for a single point, $(x, y, z)=(0,0,-1) \mathrm{m}$, on the material surface $S_{m}$. The acoustic pressures used for the calculation were taken at distances of $25 \mathrm{~mm},(x, y, z)=(0,0,-975) \mathrm{mm}$, and $50 \mathrm{~mm},(x, y, z)=(0,0,-950) \mathrm{mm}$, above the material surface.

The finite element model only represents half of the domain, making use of symmetry, see Fig. 1(b). To resemble a semi-infinite sound field, the boundary condition at the outer spherical boundary was set to a plane wave radiation condition. Preliminary simulations were carried out to determine the dimensions of the domain to ensure that reflections from this boundary will be neglectable. The model consists of 154368 quadratic tetrahedral elements, yielding a total number of 215574 degrees of freedom. The maximum overall element size was set to $0.1 \mathrm{~m}$, resulting in a minimum of 4.8 nodes/wavelength at $1500 \mathrm{~Hz}$. In a region enclosing the integration surfaces, at least 23 nodes/wavelength are present. Spatial integration of the incident and active sound intensity is performed over the integration surfaces shown in Fig. 1(b). The accuracy of the numerical spatial integration was ensured by carrying out preliminary 
simulations for different mesh sizes of a modified model, in which the boundary condition of the plane $z=-1 \mathrm{~m}$ was set to a radiation condition.

Simulations were performed for two values of the specific acoustic surface impedance $Z_{S}$, chosen such that they resemble a surface with a medium and low sound absorption coefficient. The first value is $Z_{S}=2 Z_{0}(1+i)$, and the second value equals $Z_{S}=20 Z_{0}$, where $Z_{0}=\rho_{0} c_{0} . Z_{S}$ is chosen to be independent of frequency so that variations of the resulting sound absorption coefficient curves are only caused by variations in the sound field. The simulations were carried out in the frequency range from 100 to $1500 \mathrm{~Hz}$, in steps of $25 \mathrm{~Hz}$. To analyze the accuracy of the LPW method, the exact incident sound power is compared with the estimated sound power as obtained with LPW method. The exact incident sound power is calculated by spatial integration of the incident sound intensity over surface $S_{m}$, Eq. (7). This expression was obtained analytically by mimicking the effect of the reflective wall by a mirror source,

$$
\begin{aligned}
I_{\mathrm{in}, \mathbf{n}}\left(r_{1}, r_{2}, \omega\right)= & -\frac{h|C(\omega)|^{2}}{2 Z_{0}}\left[\frac{1}{r_{1}^{3}}+\frac{1}{r_{2}^{3}}+\cos \left[k\left(r_{1}-r_{2}\right)\right]\left(\frac{r_{1}+r_{2}}{r_{1}^{2} r_{2}^{2}}\right)\right. \\
& \left.+\sin \left[k\left(r_{1}-r_{2}\right)\right]\left(\frac{r_{1}^{2}+r_{2}^{2}}{k r_{1}^{3} r_{2}^{3}}\right)\right],
\end{aligned}
$$

where $C(\omega)$ is the complex amplitude of the point source so that $P\left(r_{1}, \omega\right)$ $=\left[C(\omega) / r_{1}\right] e^{-i k r_{1}}$ for a free field, $r_{1}$ and $r_{2}$ are the respective distances to the point source and the mirror source. The estimated incident sound intensity is obtained by Eqs. (4) and (6), where $P(\mathbf{r}, \omega)$ and $U_{z}(\mathbf{r}, \omega)$ are taken from the finite element model. Both quantities are also used to calculate the active sound power flowing through surface $S^{\prime}$ by spatial integration of the active intensity in the negative $z$ direction, given by

$$
I_{\mathrm{ac}, \mathbf{n}}(\omega)=-\frac{1}{2} \operatorname{Re}\left[P(\omega) \overline{U_{z}(\omega)}\right]
$$

where the minus sign originates from the chosen direction of $\mathbf{n}$. The distribution of the exact incident intensity $I_{\text {in, }}(\omega)$ over a section of the material surface at a frequency of $1500 \mathrm{~Hz}$ is shown in Fig. 2(a). Note that this distribution must be independent of the surface impedance. Figures 2(b) and 2(c) show the estimated incident sound intensity distributions obtained by applying the LPW method at a distance $d=25 \mathrm{~mm}$ (a measurement at exactly $d=0 \mathrm{~mm}$ is not possible in practice) for $Z_{S}=2 Z_{0}(1+i)$ and $Z_{S}=20 Z_{0}$, respectively. The agreement between the estimated distributions in Figs. 2(b) and 2(c) and the exact distribution in Fig. 2(a) is very good. Instead of comparing the estimated and exact incident sound powers, we have chosen to evaluate the estimated and exact sound absorption coefficients, as these are the quantities one is ultimately interested in.

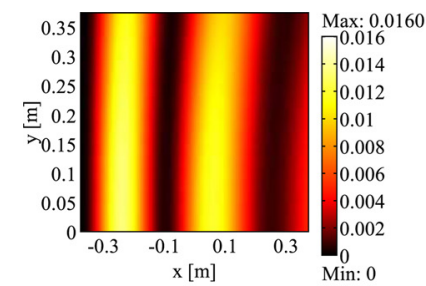

(a) Exact

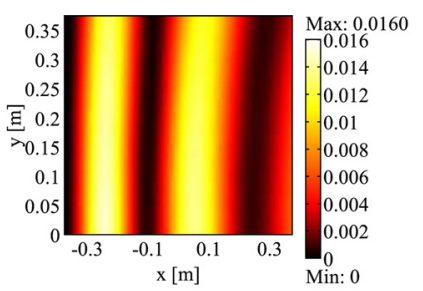

(b) Estimated, $Z_{S}=2 Z_{0}(1+i)$

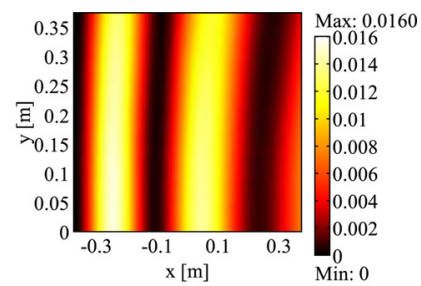

(c) Estimated, $Z_{S}=20 Z_{0}$

Fig. 2. (Color online) Distribution of the exact incident sound intensity at surface $S^{\prime}$ at $d=0 \mathrm{~mm}$, (a), and the estimated incident sound intensity as determined with the LPW method for $Z_{S}=2 Z_{0}(1+i)$ and $Z_{S}=20 Z_{0}$, (b) and (c), both at $d=25 \mathrm{~mm}$. 
Figure 3 shows the exact (normal curve) and the estimated (LPW method, dotted) curves versus frequency of the effective, area-averaged, sound absorption coefficient versus frequency for different combinations of $a$, the side length of the square integration area, and $d$, the distance. Although physically not measurable, the curves for distance $d=0 \mathrm{~mm}$ are shown to illustrate the theoretically optimal case. The other distance values $(25$ and $50 \mathrm{~mm}$ ) were chosen because they are more representative for a measurement using a $p p$ probe. With a $p u$ probe, the sound pressure and the particle

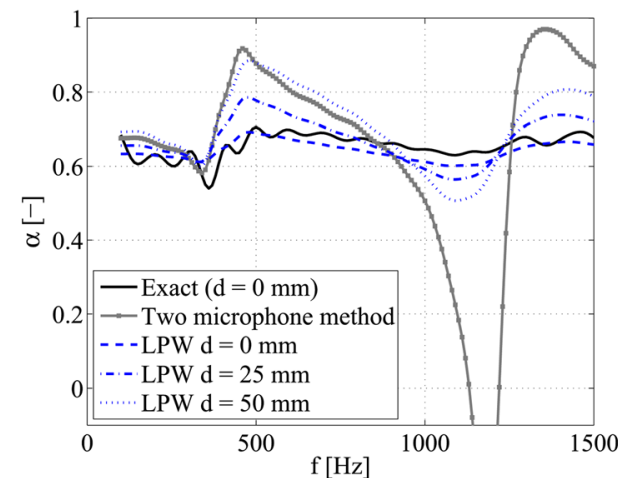

(a) $Z_{S}=2 Z_{0}(1+i), a=0.25 \mathrm{~m}$

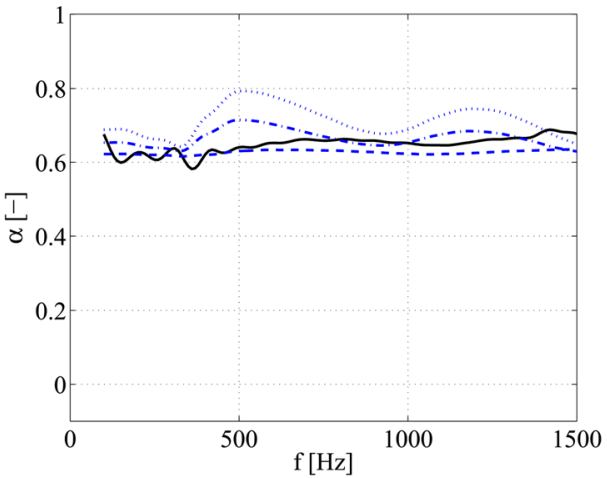

(c) $Z_{S}=2 Z_{0}(1+i), a=0.50 \mathrm{~m}$

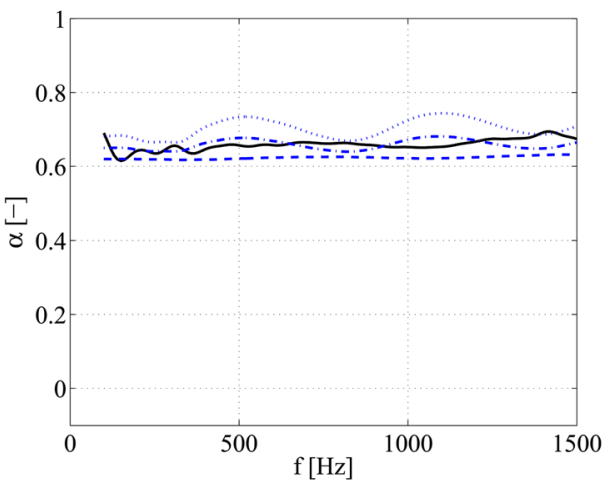

(e) $Z_{S}=2 Z_{0}(1+i), a=0.75 \mathrm{~m}$

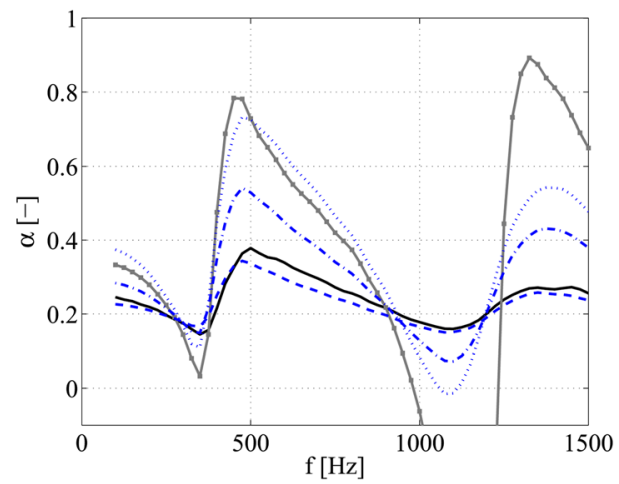

(b) $Z_{S}=20 Z_{0}, a=0.25 \mathrm{~m}$

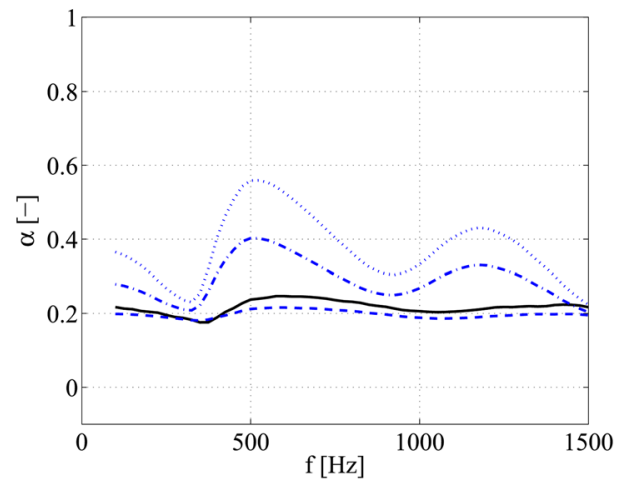

(d) $Z_{S}=20 Z_{0}, a=0.50 \mathrm{~m}$

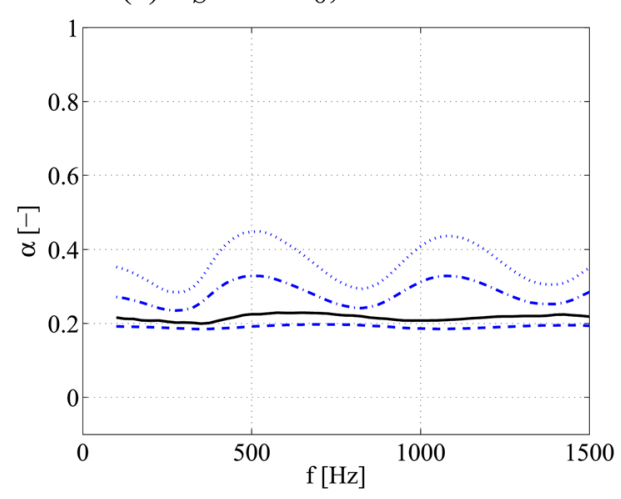

(f) $Z_{S}=20 Z_{0}, a=0.75 \mathrm{~m}$

Fig. 3. (Color online) Exact (normal curve) and estimated (LPW method, dotted) area- averaged sound absorption coefficient $\alpha$ as a function of frequency, evaluated for different integration area sizes and distances. Left column: $Z_{S}=2 Z_{0}(1+i)$. Right column: $Z_{S}=20 Z_{0}$. Both top figures also contain the curves obtained with the two-microphone method. 
velocity can be measured within a few millimeters of the surface, correspondingly the curves for $d=0 \mathrm{~mm}$ may be taken to represent such a measurement. The left column of graphs was obtained for $Z_{S}=2 Z_{0}(1+i)$, the right column for $Z_{S}=20 Z_{0}$. Both top graphs also show the effective sound absorption coefficient obtained with the twomicrophone method by Allard and Sieben. ${ }^{2}$

Figures 3(a), 3(c), and 3(e), for $Z_{S}=2 Z_{0}(1+i)$, show that the curves of the estimated effective area-averaged sound absorption coefficient obtained with the LPW method agree well with the exact curves for small distances $d$. The influence of the distance $d$ increases for decreasing surface integration area. One explanation for this observation is that propagation of acoustic energy toward or from the volume between the measurement surface and the material surface is not entirely captured as a part of the acoustic energy will pass through the circumferential area between both surfaces. This area is significant: For $d=50 \mathrm{~mm}$ and $a=0.25 \mathrm{~m}$, it equals $80 \%$ of the measurement surface area. It is expected that this increases for large incidence angles and for increasing ratios $d / a$ Therefore it is advantageous to minimize the distance $d$ between the material and measurement surface.

An increase of the surface integration area (larger value of a) leads to a smoothing of the exact curves in Fig. 3 due to area averaging of local interferences. However, this increase leads to a reduced spatial resolution if local variations of the sound absorption coefficient are investigated. For $Z_{S}=20 Z_{0}$, Figs. 3(b), 3(d), and 3(f) show that accurate estimates for the effective area-averaged sound absorption curves are only obtained when the distance $d$ is very small. A distance of less than $10 \mathrm{~mm}$ seems to be required in this case. Furthermore, the sensitivity of the effective areaaveraged sound absorption curves with respect to the distance $d$ and integration area is increased compared to the graphs for $Z_{S}=2 Z_{0}(1+i)$ in the left column. Finally, it is observed that the effective area-averaged sound absorption curves obtained with the LPW method lie close to the theoretical values of the sound absorption coefficient for purely normal incidence, being 0.62 and 0.18 , respectively.

The effective sound absorption coefficient obtained with the two-microphone method in Figs. 3(a) and 3(b) follows the LPW $d=50 \mathrm{~mm}$ curve partly. Interferences near $1200 \mathrm{~Hz}$ result in a negative effective sound absorption coefficient. Possible causes for this effect are (1) that the actual sound field is different from the sound field assumed by the two-microphone method and (2) that interference effects cannot be reduced by area averaging with a point measurement. As such, the calculated surface impedance is strongly dependent on the local sound field near the two microphones.

\section{Conclusions}

The LPW method is capable of estimating the incident sound intensity without a priori knowledge of the sound field, therefore an overall sound field model is not required. Acoustic finite element simulations for a typical case show that the accuracy of the method increases with decreasing distance to the surface of interest and with increasing surface area. The sensitivity of the accuracy on both parameters is influenced by the specific acoustic surface impedance. We conclude that the LPW method, employing area averaging, is a potentially useful technique to determine the effective areaaveraged in situ sound absorption coefficient in sound fields with multiple reflections.

\section{References and links}

${ }^{1}$ E. Brandão, E. Tijs, A. Lenzi, and H.-E. de Bree, "A comparison of three methods to calculate the surface impedance and absorption coefficient from measurements under free field or in situ conditions," Acta. Acust. Acust. 97, 1025-1033 (2011).

${ }^{2}$ J.-F. Allard and B. Sieben, "Measurements of acoustic impedance in a free field with two microphones and a spectrum analyzer," J. Acoust. Soc. Am. 77, 1617-1618 (1985).

${ }^{3}$ Y. Champoux and J. Nicolas, "Measurements of acoustic impedance in a free field at low frequencies," J. Sound Vib. 125, 313-323 (1988). 
${ }^{4}$ R. Lanoye, G. Vermeir, W. Lauriks, R. Kruse, and V. Mellert, "Measuring the free field acoustic impedance and absorption coefficient of sound absorbing materials with a combined particle velocitypressure sensor," J. Acoust. Soc. Am. 119, 2826-2831 (2006).

${ }^{5}$ K. Hirosawa, K. Takashima, H. Nakagawa, M. Kon, A. Yamamoto, and W. Lauriks, "Comparison of three measurement techniques for the normal absorption coefficient of sound absorbing materials in the free field," J. Acoust. Soc. Am. 126, 3020-3027 (2009).

${ }^{6} \mathrm{M}$. Garai, "Measurement of the sound-absorption coefficient in situ: The reflection method using periodic pseudo-random sequences of maximum length," Appl. Acoust. 39, 199-139 (1993).

${ }^{7} \mathrm{E}$. Mommertz, "Angle-dependent in-situ measurements of reflection coefficients using a subtraction technique," Appl. Acoust. 46, 251-263 (1995).

${ }^{8} \mathrm{C}$. Nocke, "In-situ acoustic impedance measurement using a free-field transfer function method," Appl. Acoust. 59, 253-264 (2000).

${ }^{9}$ Y. Takahashi, T. Otsuru, and R. Tomiku, "In situ measurements of surface impedance and absorption coefficients of porous materials using two microphones and ambient noise," Appl. Acoust. 66, 845-865 (2005).

${ }^{10}$ T. Otsuru, R. Tomiku, N. B. C. Din, N. Okamoto, and M. Murakami, "Ensemble averaged surface normal impedance of material using an in-situ technique: Preliminary study using boundary element method," J. Acoust. Soc. Am 125, 3784-3791 (2009).

${ }^{11}$ N. B. C. Din, T. Otsuru, R. Tomiku, N. Okamoto, and K. Asniawaty, "Measurement method with a pressure-velocity sensor for measuring surface normal impedance of materials using ensemble averaging: Comparison with other methods and its geometrical configuration," Acoust. Sci Tech. 33, 86-95 (2012).

${ }^{12} \mathrm{Y}$. Wijnant, E. Kuipers, and A. de Boer, "Development and application of a new method for the in-situ measurement of sound absorption," in Proceedings of ISMA 31, Leuven, Belgium (2010), Vol. 31.

${ }^{13}$ F. Fahy, Sound Intensity, 2nd ed. (E\&FN Spon, London, 1995).

${ }^{14}$ H.-E. de Bree, “An overview of microflown technologies," Acta. Acust. Acust. 89, 163-172 (2003). 